Esther Burkert

Rechtsextremismus und Geschlecht 
Beiträge zur Geschlechterforschung

Band 4 


\title{
Rechtsextremismus und Geschlecht
}

\author{
Politische Selbstverortung \\ weiblicher Auszubildender
}

Esther Burkert

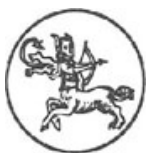

Centaurus Verlag \& Media UG 2006 


\section{Zur Autorin:}

Esther Burkert, geb. 1967, studierte Psychologie in Regensburg und promovierte als Stipendiatin der Hans-Böckler-Stiftung 2005 an der Universität Hildesheim. Sie war Mitarbeiterin unterschiedlicher Forschungsprojekte: Soziale Beziehungen in der Familie, geschlechtsspezifische Sozialisation und die Entwicklung rechtsextremer Orientierungen (Universität Hildesheim 1994-1996); Prävention und Schulentwicklung (1999, Universität Regensburg); Lernen im Netz (2000, Universität Hildesheim); Rechtsextremismus am Bau - Entwicklung von didaktischen Materialien (2001, IG BAU Frankfurt/M.). Seit 1982 ist sie in der Frauennotrufarbeit engagiert - seit 2004 arbeitet sie beim Frauennotruf Nürnberg an der Entwicklung eines Gruppenkonzeptes für schwer traumatisierte Frauen und Unterstützungsangeboten für kriegstraumatisierte ältere Frauen.

\section{Die Deutsche Bibliothek - CIP-Einheitsaufnahme}

\section{Burkert, Esther:}

Rechtsextremismus und Geschlecht : Politische Selbstverortung weiblicher Auszubildender/ Esther Burkert. -

Herbolzheim : Centaurus-Verl., 2006

(Beiträge zur Geschlechterforschung ; Bd. 4)

Zugl.: Hildesheim, Univ., Diss., 2005

ISBN 978-3-8255-0636-0

ISBN 978-3-86226-454-4 (eBook)

DOI 10.1007/978-3-86226-454-4

Erstgutachterin der Dissertation: Prof. Dr. Hede Helfrich-Hölter

Zweitgutachterinnen: Prof. Dr. Christel Hopf

Prof. Dr. Helga Bilden

\section{ISSN 1434-7415}

Alle Rechte, insbesondere das Recht der Vervielfältigung und Verbreitung sowie der Übersetzung, vorbehalten. Kein Teil des Werkes darf in irgendeiner Form (durch Fotokopie, Mikrofilm oder ein anderes Verfahren) ohne schriftliche Genehmigung des Verlages reproduziert oder unter Verwendung elektronischer Systeme verarbeitet, vervielfältigt oder verbreitet werden.

(C) CENTAURUS Verlags-GmbH. \& Co. KG, Herbolzheim 2006

Satz: Vorlage der Autorin

Umschlaggestaltung: Jasmin Morgenthaler

Umschlagabbildung unten rechts: Springerstiefel von NPD-Demonstranten.

(C) SV-Bilderdienst: Teutopress / Alle weiteren Abbildungen wurden zur

Verfügung gestellt von photocase.com. 


\section{Danksagung}

Danke an alle, die mich in so vielerlei Weise unterstützt haben,

- dass ich die Arbeit begonnen habe,

- dass ich bei der Arbeit geblieben bin,

- und dass ich auch einen Schluss gefunden habe,

und das alles meistens mit Freude.

Danke für inspirierende Gespräche und Texte, danke für interessante, bewegende, klärende und Neues eröffnende Gedanken!

Danke an die „alten“ Wissenschaftlerinnen, die den aufregenden, belebenden, manchmal steinigen, bisweilen ermüdenden, auch dann und wann schwer zu findenden Weg vor mir gegangen sind - und von denen ich so viel gelernt habe und so viel Unterstützung und Wohlwollen bekommen habe! Bedanken muss ich mich auch, dass sie ausgehalten haben, dass ich manchmal nicht so recht begeistert war, dass sie, natürlich und gut so, nicht die perfekten Vorbilder aus meinen Träumen abgegeben haben.

Und danke an die ,jungen“ WissenschaftlerInnen, die mit mir gestöhnt und geächzt, gejammert und gekichert haben und ohne die ich ebenfalls nie zum Abschluss dieser Arbeit gekommen wäre!

Vielen Dank an die jungen Frauen, die uns in ihren Interviews von sich erzählt haben - ohne ihre Bereitschaft mitzumachen, gäbe es dieses Buch nicht.

Und danke für alles Praktische, das dafür gesorgt hat, dass aus all den Gedanken ein Buch geworden ist - das Korrekturlesen, das Begutachten, das am Druck Arbeiten und alles zum Kaffee zwischendurch Einladen.

Und danke an meine Tochter, an meinen Garten und alle Freundschaft, die mich gestärkt haben, mit über lange Zeit krankem Kniegelenk, Kinderbetreuungsmanagement, Berufsalltag und Hang zu anderen Freuden und Leiden am Denken und Schreiben zu bleiben! 
Bei allen, die ich im Laufe dieses Projekts und dieser Promotion kennen und schätzen gelernt habe, möchte ich mich hier bedanken - dass mein herzlicher Dank bei ihnen ankommt, gerade jetzt, wo ich mich über das fertige Buch freue!

Bis jetzt habe ich niemanden beim Namen genannt, direkt nennen will ich zu guter Letzt doch die Hans-Böckler-Stiftung, die mich und meine Arbeit gefördert hat und die sich als Stiftung wohl weniger durch meine Worte persönlich angesprochen fühlen kann: Vielen Dank! 


\section{Inhaltsverzeichnis}

1 EINFÜHRUNG

2.1 RECHTSEXTREMISMUS - EIN DEUTSCHER SONDER-

DISKURS ODER EIN DEUTSCHES SONDERPHÄNOMEN?

$\begin{array}{lll}\text { 2.1.1 Zur Geschichte des Begriffs „Rechtsextremismus“ } & 8\end{array}$

2.1.2 Die Rechtsextremismusdefinition von Heitmeyer (1987) 11

2.1.3 Zum Verhältnis von Rechtsextremismus und Rassismus -

Unterschiedliche Konzeptionen

2.1.4 Eine Arbeitsdefinition von Rassismus

2.1.5 Prämissen und Konsequenzen unterschiedlicher Begriffsbildung: Rassismus oder Rechtsextremismus?

2.1.5.1 Demokratiefeindlichkeit oder Grundstein westlicher Demokratien? 24

2.1.5.2 Randphänomen oder vorherrschender Diskurs? 25

$\begin{array}{ll}\text { 2.1.5.3 Rolle der Wissenschaften } & 28\end{array}$

$\begin{array}{lll}\text { 2.1.6 Schlussfolgerndes Summary } & 28\end{array}$

2.2 NEUE RECHTSEXTREMISMUSFORSCHUNG IN DEUTSCHLAND: THEORETISCHE ANSÄTZE DER 90ER JAHRE UND IHRE WURZELN - EIN ÜBERBLICK 32

2.2.1 Individualisierung und Instrumentalisierung 32

$\begin{array}{lll}\text { 2.2.2 Der Jugendkulturansatz } & 39\end{array}$

2.2.3 Autoritäre Persönlichkeit heute 41

$\begin{array}{lll}\text { 2.2.4 Fazit und Kritik } & 46\end{array}$

2.3 ANREGUNGEN AUS VORURTEILSFORSCHUNG UND RASSIS-MUSTHEORIE 
2.3.1 Analyseebenen: Individuum - Gruppenbeziehungen Gesellschaft

2.3.1.1 Das Einstellungskonzept - Voraussetzungen und Grenzen $\quad 49$

2.3.1.2 Intergruppenansätze $\quad 54$

2.3.1.3 Soziale Repräsentationen $\quad 56$

2.3.2 Moderner, Aversiver und Symbolischer ,racism“

$\begin{array}{lll}\text { 2.3.3 Gegenentwürfe zu Rassismus } & 62\end{array}$

2.3.4 Zur Übertragbarkeit der Ansätze auf deutsche Verhältnisse 65

2.3.4.1 Elemente des Migrationsdiskurses in Deutschland 66

$\begin{array}{ll}\text { 2.3.4.2 Argumentationsmuster und -strukturen } & 67\end{array}$

2.3.5 Schlussfolgerndes Summary 69

3 FRAU UND RECHTSEXTREMISMUS IN THEORIE UND EMPIRIE

3.1 ANSÄTZE DER THEORIEDIKUSSION UM FRAUEN UND RECHTSEXTREMISMUS

3.1.1 Übertragung von Ansätzen aus der Jugendrechtsextremismusforschung auf Frauen

3.1.2 "gender“ im Ansatz von Siller

$\begin{array}{lll}\text { 3.1.3 Der Dominanzkulturansatz } & 75\end{array}$

$\begin{array}{lll}\text { 3.1.4 Geschlechterverhältnis bei Rommelspacher } & 80\end{array}$

3.1.5 Vergleich der Positionen von Siller und Rommelspacher 82

$\begin{array}{lll}\text { 3.1.6 Frauen in der Rassismusdebatte } & 84\end{array}$

$\begin{array}{lll}\text { 3.1.7 Verbreitete Thesen zu Frauen und Rechtsextremismus } & 84\end{array}$

3.2 EMPIRISCHE UNTERSUCHUNGEN ZUM WEIBLICHEN RECHTSEXTREMISMUS - FRAGESTELLUNGEN, ERGEBNISSE, SCHLUSSFOLGERUNGEN 
4.1 GESCHLECHT IN DEN INTERVIEWS: ÜBERPRÜFUNG DER HYPOTHESEN DER FORSCHUNG ZUM „WEIBLICHEN" UND ZUM „MÄNNLICHEN" RECHTSEXTREMISMUS

4.2 VERKNÜPFUNG THEORETISCHER PERSPEKTIVEN RECHTSEXTREMISMUSFORSCHUNG UND

ANGLOAMERIKANISCHE RASSISMUSDISKUSSION:

KONKRETE FRAGEN

5 METHODISCHES VORGEHEN

5.2.1 Konstruktion der Interviewleitfäden

5.2.2 Zwischen Geschlechterinsensibilität und Geschlechterdichotomie - Methodische Fallstricke 
6.1 BESTIMMUNGSPUNKTE ZUR BESCHREIBUNG DER STICHPROBE

6.1.1 Sozioökonomischer Hintergrund der Befragten

6.1.2 Desinteresse an Politik

6.1.3 Auseinandersetzung mit dem historischen

Nationalsozialismus

\subsection{ARGUMENTIEREN FRAUEN „FRAUENSPEZIFISCH”? - ÜBERPRÜFUNG DER ARGUMENTATIONSSTRUKTUREN IN DEN INTERVIEWS}

6.2.1 Bedrohungsgefühle durch sexualisierte Gewalt und ethnozentrische Haltungen bei Frauen

6.2.2 Angst vor Kriminalität als Einfallstor für rechtsextreme Orientierungen von Frauen?

6.2.3 Hoher Konventionalismus

6.2.4 Gewaltbedürfnisse von Frauen - an Männer und Institutionen delegiert?

6.2.5 Ökonomismus - Sprechen Frauen nicht über Arbeitsplätze und Sozialleistungen?

6.2.6 Zusammenfassendes Fazit

6.3 EINZELFALLANALYSEN

6.3.1 Zu den Inhalten und zur Struktur des Migrationsdiskurses in Deutschland - Unterscheidungskriterien

6.3.2 Unterschiedliche Selbstverortungen im Migrationsdiskurs von der generalisierten Diskriminierung zum Widerspruch 
6.3.2.1 Gruppe 1: Generalisierte Diskriminierung $\quad 177$

6.3.2.2 Gruppe 2: Differenzierende Diskriminierung 200 6.3.2.3 Gruppe 3: Betonung des Prinzips Leistung vor Staats-
angehörigkeit

6.3.2.4 Gruppe 4: Widerspruch zu Diskriminierung 213

6.3.2.5 Gruppe 5: Ambivalenz zwischen politischer Diskrimnierung und $\begin{array}{ll}\text { privaten Beziehungen } & 219\end{array}$

6.3.3 Zusammenfassendes Fazit $\quad 225$

6.3.3.1 Hauptprinzip: Feindbild mit flexiblen Grenzen 226

6.3.3.2 „Wissen“ über die „Anderen“ und die Gestaltung konkreter Beziehungen 231

6.3.4 „Gender“ in den Einzelfallanalysen 235

7 GESCHLECHTERKONSTRUKTION UND ETHNISIERUNG 239

7.1 Konstruktion von Geschlecht im Forschungsprozess 239

7.2 Zentrale Argumentationsmuster: Dichotomie und Alteroreferenzialität

7.3 Geschlecht, politische Orientierung und politisches Handeln 244

8 LITERATURVERZEICHNIS 246

9 VERZEICHNIS DER TABELLEN 267 